\title{
Transmission Dynamics of a Two-City SIR Epidemic Model with Transport-Related Infections
}

\author{
Yao Chen, ${ }^{1,2,3}$ Mei Yan, ${ }^{2}$ and Zhongyi Xiang ${ }^{1,2}$ \\ ${ }^{1}$ Key Laboratory of Biologic Resources Protection and Utilization of Hubei Province, Enshi, Hubei 445000, China \\ ${ }^{2}$ Department of Mathematics, Hubei University for Nationalities, Enshi, Hubei 445000, China \\ ${ }^{3}$ Science and Technology College of Hubei University for Nationalities, Enshi, Hubei 445000, China
}

Correspondence should be addressed to Zhongyi Xiang; zhyxiang260@yahoo.com.cn

Received 20 April 2013; Accepted 12 December 2013; Published 23 January 2014

Academic Editor: Xinyu Song

Copyright (c) 2014 Yao Chen et al. This is an open access article distributed under the Creative Commons Attribution License, which permits unrestricted use, distribution, and reproduction in any medium, provided the original work is properly cited.

\begin{abstract}
A two-city SIR epidemic model with transport-related infections is proposed. Some good analytical results are given for this model. If the basic reproduction number $\Re_{0 \gamma} \leq 1$, there exists a disease-free equilibrium which is globally asymptotically stable. There exists an endemic equilibrium which is locally asymptotically stable if the basic reproduction number $\mathfrak{R}_{0 \gamma}>1$. We also show the permanence of this SIR model. In addition, sufficient conditions are established for global asymptotic stability of the endemic equilibrium.
\end{abstract}

\section{Introduction}

Epidemiology is the study of the spread of disease in time and space, aiming at tracing factors that give rise to their occurrence. Since Kermark and Mckendrick in [1] built up a system to study epidemiology in 1927, the concept of "compartment modeling" is widely used until now. From then on, many great epidemic models are proposed and researched [2-5], which assume that population lives in the fixed region, without travel. However, in fact, people usually travel among different regions; thus models involving dispersal are indispensable. To control the spread of an infectious disease, we have to know how the growth and spread of the disease affect its outbreak. And there are many factors that lead to the dynamics of an infectious disease of humans, such as human behavior as population dislocations, living styles, sexual practices, and rising international travel. On the other hand, climate change enables diseases and vectors to expand their range. Since the first AIDS case was reported in the United States in June 1981, the number of cases and deaths among persons with AIDS increased rapidly during the 1980s followed by substantial declines in new cases and deaths in the late 1990s. In 2003, SARS began in Guangdong province of China; however, it broke out at last in almost all parts of China and some other cities in the world due to dispersal [6]. Recently, some epidemic models have been proposed to understand the spread dynamics of infectious disease.

Ahmed et al. in [7] introduced a model with travel between populations. In addition, Sattenspiel and Herring considered the same type of model but applied it to travel between populations in the Canadian subarctic, which can be thought of as a closed population where travel is easily quantified [8]. Ding et al. [9] and Sattenspiel et al. [10,11] have also discussed other models for the spread of a disease among two patches and $n$ patches. In [12], Wang and Mulone studied an SIS model with standard incidence rate on population dispersal among $n$ patches. Wang and Zhao [13] proposed an SEIR epidemic model, assuming that the susceptible and exposed individuals have constant immigration rates. What is more, Wang and Zhao [14] formulated a general SEIRS for multispecies on multipatches, and the role of quarantine in the form of travel restriction was discussed.

All these investigations ignore the possibility for the individuals to become infective during travel. In paper [15], Allen et al. have proposed the following SIS epidemic model to 
understand the effect of transport-related infection on disease spread for the first time:

$$
\begin{gathered}
\dot{S}_{1}=a-\frac{\beta S_{1} I_{1}}{S_{1}+I_{1}}-b S_{1}+d I_{1}-\alpha S_{1}+\alpha S_{2}-\frac{\gamma \alpha S_{2} I_{2}}{S_{2}+I_{2}}, \\
\dot{I}_{1}=\frac{\beta S_{1} I_{1}}{S_{1}+I_{1}}-(c+d+\alpha) I_{1}+\alpha I_{2}+\frac{\gamma \alpha S_{2} I_{2}}{S_{2}+I_{2}}, \\
\dot{S}_{2}=a-\frac{\beta S_{2} I_{2}}{S_{2}+I_{2}}-b S_{2}+d I_{2}-\alpha S_{2}+\alpha S_{1}-\frac{\gamma \alpha S_{1} I_{1}}{S_{1}+I_{1}}, \\
\dot{I}_{2}=\frac{\beta S_{2} I_{2}}{S_{2}+I_{2}}-(c+d+\alpha) I_{2}+\alpha I_{1}+\frac{\gamma \alpha S_{1} I_{1}}{S_{1}+I_{1}} .
\end{gathered}
$$

For many diseases (e.g., influenza, measles, chickenpox, etc.), after recovery, the individuals have immunity to the disease. Thus, an SIR or SIRS model is more suitable for this kind of disease. In this paper, we will study the effect of transport-related infection. Our results show that transportrelated infection can make the disease endemic even if both the isolated regions are disease free.

We consider a model with state variables $S_{i}, I_{i}, R_{i}$ that represent the number of susceptible, infected, and removed individuals in city $i(i=1,2)$, respectively. The basic assumptions underlying the dynamics of the system are as follows.

(i) We assume that both cities are identical.

(ii) All newborns, denoted by $a$, join into the susceptible class per unit time.

(iii) Natural death rate for susceptible, infected, and removed individuals is a constant per capita rate $b$.

(iv) Disease is transmitted with the standard form incidence rate $\beta S_{i} I_{i} / S_{i}+I_{i}+R_{i}, i=1,2$ within city $i$. The transmission rate within a city is a constant $\beta$.

(v) We may assume that a susceptible individual goes into the infected part after infection.

(vi) Susceptible, infected, and removed individuals of every city $i$ leave for city $j(i \neq j, i, j=1,2)$ at a per capita rate $\alpha$. We assume that two cities are connected by the direct transport such as airplanes or trains.

(vii) When the individuals in city $i$ travel to city $j$, disease is transmitted with the incidence rate $\gamma\left(\alpha S_{i}\right)\left(\alpha I_{i}\right) / \alpha S_{i}+$ $\alpha I_{i}=\gamma \alpha S_{i} I_{i} / S_{i}+I_{i}+R_{i}, i=1,2$ with a transmission rate $\gamma \alpha$.

(viii) The rate constant for recovery is denoted by $d$, and the per capita mortality rate for infected individual is $c$. Since this includes both natural and disease induced mortality, we have $c>b$.

(ix) We suppose that individuals who are traveling do not give birth and do not take death. Further we assume that removed individuals do not lose immunity during travel.
These assumptions lead to a model of the following form:

$$
\begin{gathered}
\dot{S}_{1}=a-\frac{\beta S_{1} I_{1}}{S_{1}+I_{1}+R_{1}}-b S_{1}-\alpha S_{1}+\alpha S_{2}-\frac{\gamma \alpha S_{2} I_{2}}{S_{2}+I_{2}+R_{2}}, \\
\dot{I}_{1}=\frac{\beta S_{1} I_{1}}{S_{1}+I_{1}+R_{1}}-(c+\alpha+b+d) I_{1}+\alpha I_{2}+\frac{\gamma \alpha S_{2} I_{2}}{S_{2}+I_{2}+R_{2}}, \\
\dot{R}_{1}=d I_{1}-b R_{1}-\alpha R_{1}+\alpha R_{2}, \\
\dot{S}_{2}=a-\frac{\beta S_{2} I_{2}}{S_{2}+I_{2}}-b S_{2}-\alpha S_{2}+\alpha S_{1}-\frac{\gamma \alpha S_{1} I_{1}}{S_{1}+I_{1}+R_{1}}, \\
\frac{\beta S_{2} I_{2}}{S_{2}+I_{2}+R_{2}}-(c+\alpha+b+d) I_{2}+\alpha I_{1}+\frac{\gamma \alpha S_{1} I_{1}}{S_{1}+I_{1}+R_{1}}, \\
\dot{R}_{2}=d I_{2}-b R_{2}-\alpha R_{2}+\alpha R_{1} .
\end{gathered}
$$

From the biological point of view, the term $\alpha S_{i}$ represents the susceptible leaving city $i$ and $\gamma \alpha S_{i} I_{i} /\left(S_{i}+I_{i}+R_{i}\right)$ denote individuals in $\alpha S_{i}$ becoming infected during travel from city $i$ to $j$. Hence, $\alpha S_{i}-\gamma \alpha S_{i} I_{i} /\left(S_{i}+I_{i}+R_{i}\right)$ should be nonnegative. Therefore, we always suppose $0 \leq \gamma \leq 1$ in the following discussion.

The paper is organized as follows. In next section, we will research the existence of equilibria and their local stability. In Section 3, we will discuss permanence of the SIR model and some sufficient conditions for global stability of equilibrium in Section 4. In the final section, we will discuss our results and give some numerical simulations.

\section{Local Stability}

The assumption that both cities are identical, that is, demographic parameters are the same for each city, has enabled us to obtain an analytic expression for the equilibria. It is easy to check that system (2) has a disease-free equilibrium $E_{0}\left(S^{0}, 0,0, S^{0}, 0,0\right)$ for all parameter values, where $S^{0}=a / b$. According to the concept of next generation matrix in $\mathrm{Li}$ et al. [16] and reproduction number presented in Liu et al. [17], we can define

$$
F=\left(\begin{array}{cccccc}
0 & 0 & 0 & 0 & 0 & 0 \\
0 & \beta & 0 & 0 & \gamma \alpha & 0 \\
0 & 0 & 0 & 0 & 0 & 0 \\
0 & 0 & 0 & 0 & 0 & 0 \\
0 & \gamma \alpha & 0 & 0 & \beta & 0 \\
0 & 0 & 0 & 0 & 0 & 0
\end{array}\right),
$$

V

$$
=\left(\begin{array}{cccccc}
b+\alpha & \beta & 0 & -\alpha & \gamma \alpha & 0 \\
0 & c+\alpha+b+d & 0 & 0 & -\alpha & 0 \\
0 & -d & b+\alpha & 0 & 0 & -\alpha \\
-\alpha & \gamma \alpha & 0 & b+\alpha & \beta & 0 \\
0 & -\alpha & 0 & 0 & c+\alpha+b+d & 0 \\
0 & 0 & -\alpha & 0 & -d & b+\alpha
\end{array}\right) .
$$

Hence the reproduction number for system (2) is

$$
\mathfrak{R}_{0 \gamma}=\rho\left(F V^{-1}\right)=\frac{\beta+\gamma \alpha}{b+d+c} .
$$


Here $\rho\left(F V^{-1}\right)$ represents the spectral radius of the matrix $F V^{-1}$. When $\Re_{0 \gamma}>1$, system (2) has unique positive equilibrium $E_{+}\left(S^{*}, I^{*}, R^{*}, S^{*}, I^{*}, R^{*}\right)$, where

$$
\begin{gathered}
I^{*}=\frac{a\left(\Re_{0 \gamma}-1\right)}{\gamma \alpha+\beta-c}, \\
S^{*}=\frac{b+d}{b\left(\Re_{0 \gamma}-1\right)} I^{*},
\end{gathered}
$$

$$
R^{*}=\frac{d}{b} I^{*} .
$$

The Jacobian matrix for the right hand of system (2) is given by

$$
J=\left(\begin{array}{ll}
A_{1} & B_{1} \\
B_{2} & A_{2}
\end{array}\right),
$$

where

$$
A_{1}=\left(\begin{array}{ccc}
-\frac{\beta I_{1}}{S_{1}+I_{1}+R_{1}}+\frac{\beta S_{1} I_{1}}{\left(S_{1}+I_{1}+R_{1}\right)^{2}}-b-\alpha & -\frac{\beta S_{1}}{S_{1}+I_{1}+R_{1}}+\frac{\beta S_{1} I_{1}}{\left(S_{1}+I_{1}+R_{1}\right)^{2}} & \frac{\beta S_{1} I_{1}}{\left(S_{1}+I_{1}+R_{1}\right)^{2}} \\
\frac{\beta I_{1}}{S_{1}+I_{1}+R_{1}}-\frac{\beta S_{1} I_{1}}{\left(S_{1}+I_{1}+R_{1}\right)^{2}} & \frac{\beta S_{1}}{S_{1}+I_{1}+R_{1}}-\frac{\beta S_{1} I_{1}}{\left(S_{1}+I_{1}+R_{1}\right)^{2}}-c-\alpha-b-d-\frac{\beta S_{1} I_{1}}{\left(S_{1}+I_{1}+R_{1}\right)^{2}} \\
0 & d & -b-\alpha
\end{array}\right),
$$

$$
B_{1}=\left(\begin{array}{ccc}
\alpha-\frac{\gamma \alpha I_{2}}{S_{2}+I_{2}+R_{2}}+\frac{\gamma \alpha S_{2} I_{2}}{\left(S_{2}+I_{2}+R_{2}\right)^{2}} & -\frac{\gamma \alpha S_{2}}{S_{2}+I_{2}+R_{2}}+\frac{\gamma \alpha S_{2} I_{2}}{\left(S_{2}+I_{2}+R_{2}\right)^{2}} & \frac{\gamma \alpha S_{2} I_{2}}{\left(S_{2}+I_{2}+R_{2}\right)^{2}} \\
\frac{\gamma \alpha I_{2}}{S_{2}+I_{2}+R_{2}}-\frac{\gamma \alpha S_{2} I_{2}}{\left(S_{2}+I_{2}+R_{2}\right)^{2}} & \alpha+\frac{\gamma \alpha S_{2}}{S_{2}+I_{2}+R_{2}}-\frac{\gamma \alpha S_{2} I_{2}}{\left(S_{2}+I_{2}+R_{2}\right)^{2}} & -\frac{\gamma \alpha S_{2} I_{2}}{\left(S_{2}+I_{2}+R_{2}\right)^{2}} \\
0 & 0 & \alpha
\end{array}\right),
$$

$$
B_{2}=\left(\begin{array}{ccc}
\alpha-\frac{\gamma \alpha I_{1}}{S_{1}+I_{1}+R_{1}}+\frac{\gamma \alpha S_{1} I_{1}}{\left(S_{1}+I_{1}+R_{1}\right)^{2}} & -\frac{\gamma \alpha S_{1}}{S_{1}+I_{1}+R_{1}}+\frac{\gamma \alpha S_{1} I_{1}}{\left(S_{1}+I_{1}+R_{1}\right)^{2}} & \frac{\gamma \alpha S_{1} I_{1}}{\left(S_{1}+I_{1}+R_{1}\right)^{2}} \\
\frac{\gamma \alpha I_{1}}{S_{1}+I_{1}+R_{1}}-\frac{\gamma \alpha S_{1} I_{1}}{\left(S_{1}+I_{1}+R_{1}\right)^{2}} & \alpha+\frac{\gamma \alpha S_{1}}{S_{1}+I_{1}+R_{1}}-\frac{\gamma \alpha S_{1} I_{1}}{\left(S_{1}+I_{1}+R_{1}\right)^{2}} & -\frac{\gamma \alpha S_{1} I_{1}}{\left(S_{1}+I_{1}+R_{1}\right)^{2}} \\
0 & 0 & \alpha
\end{array}\right),
$$

$$
A_{2}=\left(\begin{array}{ccc}
-\frac{\beta I_{2}}{S_{2}+I_{2}+R_{2}}+\frac{\beta S_{2} I_{2}}{\left(S_{2}+I_{2}+R_{2}\right)^{2}}-b-\alpha & -\frac{\beta S_{2}}{S_{2}+I_{2}+R_{2}}+\frac{\beta S_{2} I_{2}}{\left(S_{2}+I_{2}+R_{2}\right)^{2}} & \frac{\beta S_{2} I_{2}}{\left(S_{2}+I_{2}+R_{2}\right)^{2}} \\
\frac{\beta I_{2}}{S_{2}+I_{2}+R_{2}}-\frac{\beta S_{2} I_{2}}{\left(S_{2}+I_{2}+R_{2}\right)^{2}} & \frac{\beta S_{2}}{S_{2}+I_{2}+R_{2}}-\frac{\beta S_{2} I_{2}}{\left(S_{2}+I_{2}+R_{2}\right)^{2}}-c-\alpha-b-d-\frac{\beta S_{2} I_{2}}{\left(S_{2}+I_{2}+R_{2}\right)^{2}} \\
0 & d & -b-\alpha
\end{array}\right) .
$$

Firstly, we study stability of the disease-free equilibrium $E_{0}$.

Theorem 1. If $\Re_{0 \gamma}<1$, then $E_{0}$ is locally asymptotically stable, and if $\Re_{0 \gamma}>1$, then $E_{0}$ is unstable.
Proof. Evaluating (6)-(10) at $E_{0}$, we have the following Jacobian matrix:

$$
J\left(E_{0}\right)=\left(\begin{array}{ll}
A & B \\
B & A
\end{array}\right),
$$


where

$$
\begin{gathered}
A=\left(\begin{array}{ccc}
-b-\alpha & -\beta & 0 \\
0 & \beta-c-\alpha-b-d & 0 \\
0 & d & -b-\alpha
\end{array}\right), \\
B=\left(\begin{array}{ccc}
\alpha & -\gamma \alpha & 0 \\
0 & \alpha+\gamma \alpha & 0 \\
0 & 0 & \alpha
\end{array}\right) .
\end{gathered}
$$

By Allen et al. [15], the eigenvalues of $J\left(E_{0}\right)$ are identical to those of $A+B$ and $A-B$ since

$$
\begin{gathered}
A+B=\left(\begin{array}{ccc}
-b & -\beta-\gamma \alpha & 0 \\
0 & \beta-c-b-d+\gamma \alpha & 0 \\
0 & d & -b
\end{array}\right), \\
A-B=\left(\begin{array}{ccc}
-b-2 \alpha & -\beta+\gamma \alpha & 0 \\
0 & \beta-c-b-d-(2+\gamma) \alpha & 0 \\
0 & d & -b-2 \alpha
\end{array}\right) .
\end{gathered}
$$

The eigenvalues of $A+B$ are $\lambda_{1}=-b, \lambda_{2}=-b, \lambda_{3}=\beta-$ $c-b-d+\gamma \alpha$ and the eigenvalues of $A-B$ are $\lambda_{1}=-b-2 \alpha$, $\lambda_{2}=-b-2 \alpha, \lambda_{3}=\beta-c-b-d-2 \alpha-\gamma \alpha$. By $\Re_{0 \gamma}<1$, we have $\beta+\gamma \alpha<b+d+c$; we can conclude that all six eigenvalues of $J\left(E_{0}\right)$ are negative. When $\Re_{0 \gamma}>1$, we have $\beta+\gamma \alpha>b+d+c$ which implies $\lambda_{3}>0$, so we obtain that at least one eigenvalue of $J\left(E_{0}\right)$ is positive. Hence $E_{0}$ is locally asymptotically stable if $\mathfrak{R}_{0 \gamma}<1$ and $E_{0}$ is unstable if $\mathfrak{R}_{0 \gamma}>1$. This completes the proof.

Next, we research the stability of $E_{+}$. Evaluating (6)-(10) at $E_{+}$and using (5), we have the following Jacobian matrix for $E_{+}$:

$$
J\left(E_{+}\right)=\left(\begin{array}{cc}
A & B \\
B & A
\end{array}\right)
$$

where

A

$$
=\left(\begin{array}{ccc}
-\frac{\beta b}{b+d}\left(1-\frac{1}{\Re_{0 \gamma}}\right)^{2}-b-\alpha & -\frac{\beta\left(d \Re_{0 \gamma}+b\right)}{(b+d) \Re_{0 \gamma}^{2}} & \frac{b \beta\left(\Re_{0 \gamma}-1\right)}{(b+d) \Re_{0 \gamma}^{2}} \\
\frac{\beta b}{b+d}\left(1-\frac{1}{\mathfrak{R}_{0 \gamma}}\right)^{2} & \frac{\beta\left(d \Re_{0 \gamma}+b\right)}{(b+d) \Re_{0 \gamma}^{2}}-(c+\alpha+b+d) & -\frac{b\left(\Re_{0 \gamma}-1\right)}{(b+d) \Re_{0 \gamma}^{2}}
\end{array}\right),
$$

$B$

$$
=\left(\begin{array}{ccc}
\alpha-\frac{b \gamma \alpha}{b+d}\left(1-\frac{1}{\mathfrak{R}_{0 \gamma}}\right)^{2} & -\frac{\gamma \alpha\left(d \Re_{0 \gamma}+b\right)}{(b+d) \Re_{0 \gamma}^{2}} & \frac{b \gamma \alpha\left(\Re_{0 \gamma}-1\right)}{(b+d) \mathfrak{R}_{0 \gamma}^{2}} \\
\frac{b \gamma \alpha}{b+d}\left(1-\frac{1}{\mathfrak{R}_{0 \gamma}}\right)^{2} & \alpha+\frac{\gamma \alpha\left(d \Re_{0 \gamma}+b\right)}{(b+d) \Re_{0 \gamma}^{2}} & -\frac{b \gamma \alpha\left(\Re_{0 \gamma}-1\right)}{(b+d) \mathfrak{R}_{0 \gamma}^{2}} \\
0 & 0 & \alpha
\end{array}\right) .
$$

Similar to the proof of Theorem 1, to calculate the eigenvalues of $J\left(E_{+}\right)$is equivalent to calculating the eigenvalues of matrix $A+B$ and $A-B$, where

$$
\begin{gathered}
A+B=\left(\begin{array}{ccc}
-\frac{b(\beta+\gamma \alpha)}{b+d}\left(1-\frac{1}{\mathfrak{R}_{0 \gamma}}\right)^{2}-b & -\frac{(\beta+\gamma \alpha)\left(d \mathfrak{R}_{0 \gamma}+b\right)}{(b+d) \mathfrak{R}_{0 \gamma}^{2}} & \frac{b(\beta+\gamma \alpha)\left(\mathfrak{R}_{0 \gamma}-1\right)}{(b+d) \mathfrak{R}_{0 \gamma}^{2}} \\
\frac{b(\beta+\gamma \alpha)}{b+d}\left(1-\frac{1}{\mathfrak{R}_{0 \gamma}}\right)^{2} & \frac{(\beta+\gamma \alpha)\left(d \mathfrak{R}_{0 \gamma}+b\right)}{(b+d) \mathfrak{R}_{0 \gamma}^{2}}-(c+b+d) & -\frac{b(\beta+\gamma \alpha)\left(\mathfrak{R}_{0 \gamma}-1\right)}{(b+d) \mathfrak{R}_{0 \gamma}^{2}}
\end{array}\right), \\
0
\end{gathered}
$$

However, different from the case for $E_{0}$, the eigenvalues of matrices $A+B$ and $A-B$ cannot be calculated explicitly. We will use the Routh-Hurwitz Theorem to study the stability of $E_{+}$. Note that $A+B$ and $A-B$ have the same form as follows:

$$
J=\left(\begin{array}{ccc}
a_{11} & a_{12} & a_{13} \\
a_{21} & a_{22} & a_{23} \\
0 & a_{32} & a_{33}
\end{array}\right)
$$

The characteristic polynomial of matrix $J$ is $\lambda^{3}+A_{1} \lambda^{2}+$ $A_{2} \lambda+A_{3}=0$, where $A_{1}=-\operatorname{tr}(J), A_{2}=J_{1}+J_{2}+J_{3}$, and $A_{3}=-\operatorname{det}(J)$ and $J_{1}=a_{11} a_{22}-a_{21} a_{12}, J_{2}=a_{11} a_{33}$, and
$J_{3}=a_{22} a_{33}-a_{23} a_{32}$. For convenience, we state the RouthHurwitz Theorem for above matrix.

Lemma 2. $J$ is stable (i.e., each eigenvalue of $J$ has negative real part) if and only if the following conditions hold:

(i) $A_{1}>0$,

(ii) $A_{3}>0$,

(iii) $A_{1} A_{2}-A_{3}>0$.

$E_{+}$.
Using Lemma 2, we have the following stability result for 
Theorem 3. If $\mathfrak{R}_{0 \gamma}>1$, then $E_{+}$is locally asymptotically stable.

Proof. Consider the matrices $A$ and $B$ in $J\left(E_{+}\right)$, the Jacobian matrix of system (2) at $E_{+}$. It suffices to check that both $A+B$ and $A-B$ satisfy the conditions in Lemma 2. Firstly, we check them for $A+B$ as the following three steps. For simplification, we will refer the entries of $A+B$ as $a_{i j}, i, j=1,2,3$.

(i) $A_{1}>0$. Obviously, $a_{11}<0$ and $a_{33}<0$. By (4),

$$
\begin{aligned}
a_{22}= & \frac{(\beta+\gamma \alpha)\left(d \mathfrak{R}_{0 \gamma}+b\right)}{(b+d) \mathfrak{R}_{0 \gamma}^{2}}-(c+b+d) \\
= & \left((\beta+\gamma \alpha)\left(d \mathfrak{R}_{0 \gamma}+b \Re_{0 \gamma}\right)\right. \\
& \left.+(\beta+\gamma \alpha)\left(b-b \mathfrak{R}_{0 \gamma}\right)\right) \times\left((b+d) \mathfrak{R}_{0 \gamma}^{2}\right)^{-1} \\
& -(c+b+d) \\
= & \frac{b(\beta+\gamma \alpha)\left(1-\mathfrak{R}_{0 \gamma}\right)}{(b+d) \mathfrak{R}_{0 \gamma}^{2}} .
\end{aligned}
$$

Since $\mathfrak{R}_{0 \gamma}>1$, we have $a_{22}<0$. Thus $A_{1}=-\operatorname{tr}(A+B)=$ $-\left(a_{11}+a_{22}+a_{33}\right)>0$.

(ii) $J_{i}>0, i=1,2,3$. Obviously, $a_{i i}<0, i=1,2,3, a_{12}<$ $0, a_{21}>0, a_{23}<0, a_{32}>0$. So $J_{1}=a_{11} a_{22}-a_{21} a_{12}>0$, $J_{2}=a_{11} a_{33}>0, J_{3}=a_{22} a_{33}-a_{23} a_{32}>0$. obtain

(iii) $A_{3}>0, A_{1} A_{2}-A_{3}>0$. Since $a_{21}>0, J_{3}>0$, we

$$
\begin{aligned}
A_{3} & =-\operatorname{det}(A+B) \\
& =-\left(a_{11} a_{22} a_{33}+a_{13} a_{21} a_{32}-a_{12} a_{21} a_{33}-a_{11} a_{23} a_{32}\right) \\
& =b J_{3}+b(c+b+d) a_{21}>0 .
\end{aligned}
$$

We also have

$$
\begin{aligned}
A_{1} A_{2}- & A_{3} \\
= & -\left(a_{11}+a_{22}+a_{33}\right)\left(J_{1}+J_{2}+J_{3}\right)+\operatorname{det}(A+B) \\
= & -a_{11}\left(J_{1}+J_{2}\right)-a_{22}\left(J_{1}+J_{2}+J_{3}\right)-a_{33}\left(J_{2}+J_{3}\right) \\
& +a_{13} a_{21} a_{32}-a_{11} a_{22} a_{33} .
\end{aligned}
$$

Thus, $A_{1} A_{2}-A_{3}>0$ by the fact that $a_{i i}<0, J_{i}>0, i=1,2,3$, and $a_{13}>0, a_{21}>0, a_{32}>0$. Therefore, by Lemma $2, A+B$ is stable. Next we check $A-B$ as follows. For convenience, we also refer the entries of $A-B$ as $a_{i j}, i, j=1,2,3$.

(i) $A_{1}>0$. Obviously, $a_{33}<0$. Since $0<\left(1-1 / \Re_{0 \gamma}\right)^{2}<1$ when $\mathfrak{R}_{0 \gamma}>1$ and also noting that $0 \leq \gamma \leq 1$, we have

$$
a_{11}=-\frac{b(\beta-\gamma \alpha)}{b+d}\left(1-\frac{1}{\mathfrak{R}_{0 \gamma}}\right)^{2}-b-2 \alpha
$$

$$
\begin{aligned}
= & -\frac{b \beta}{b+d}\left(1-\frac{1}{\mathfrak{R}_{0 \gamma}}\right)^{2}-b \\
& -\left(2-\frac{b \gamma}{b+d}\left(1-\frac{1}{\mathfrak{R}_{0 \gamma}}\right)^{2}\right) \alpha<0
\end{aligned}
$$

the same to (18), one has

$$
\begin{aligned}
a_{22} & =\frac{(\beta-\gamma \alpha)\left(d \Re_{0 \gamma}+b\right)}{(b+d) \mathfrak{R}_{0 \gamma}^{2}}-(c+b+d+2 \alpha) \\
& \leq \frac{(\beta+\gamma \alpha)\left(d \mathfrak{R}_{0 \gamma}+b\right)}{(b+d) \mathfrak{R}_{0 \gamma}^{2}}-(c+b+d)<0 .
\end{aligned}
$$

Thus, $A_{1}=-\operatorname{tr}(A-B)=-\left(a_{11}+a_{22}+a_{33}\right)>0$.

(ii) $J_{i}>0, i=1,2,3$. Since $a_{i i}<0, i=1,2,3$. Obviously $J_{2}=a_{11} a_{33}>0$. For $J_{1}$, we have

$$
\begin{aligned}
J_{1}= & a_{11} a_{22}-a_{21} a_{12}=\left(a_{11}+a_{21}\right) a_{22}-\left(a_{12}+a_{22}\right) a_{21} \\
= & (b+2 \alpha)(c+b+d+2 \alpha) \\
& -\frac{(b+2 \alpha)(\beta-\gamma \alpha)\left(d \Re_{0 \gamma}+b\right)}{(b+d) \mathfrak{R}_{0 \gamma}^{2}} \\
& +\frac{b(c+b+d+2 \alpha)(\beta-\gamma \alpha)}{b+d}\left(1-\frac{1}{\mathfrak{R}_{0 \gamma}}\right)^{2} .
\end{aligned}
$$

$J_{1}>0$ can be shown as the following two cases.

Case $1(\beta-\gamma \alpha>0)$. By (4), we obtain that

$$
b+d+c=(\beta+\gamma \alpha) \frac{1}{\mathfrak{R}_{0 \gamma}}=(\beta-\gamma \alpha+2 \gamma \alpha) \frac{1}{\mathfrak{R}_{0 \gamma}} .
$$

Thus, $J_{1}$ can be rewritten as

$$
\begin{aligned}
J_{1}> & (b+2 \alpha)\left[2 \alpha+(\beta-\gamma \alpha+2 \gamma \alpha) \frac{1}{\mathfrak{R}_{0 \gamma}}\right] \\
& -(b+2 \alpha)(\beta-\gamma \alpha) \frac{1}{\mathfrak{R}_{0 \gamma}} \\
& +\frac{b(c+b+d+2 \alpha)(\beta-\gamma \alpha)}{b+d}\left(1-\frac{1}{\mathfrak{R}_{0 \gamma}}\right)^{2} \\
= & (b+2 \alpha)\left(2 \alpha+2 \gamma \alpha \frac{1}{\mathfrak{R}_{0 \gamma}}\right) \\
& +\frac{b(c+b+d+2 \alpha)(\beta-\gamma \alpha)}{b+d}\left(1-\frac{1}{\mathfrak{R}_{0 \gamma}}\right)^{2} .
\end{aligned}
$$

Since $\mathfrak{R}_{0 \gamma}>1$, it is clear that $J_{1}>0$. 
Case $2(\beta-\gamma \alpha \leq 0)$. According to $\mathfrak{R}_{0 \gamma}>1$, we have

$$
\begin{aligned}
J_{1}= & (b+2 \alpha)(c+b+d+2 \alpha) \\
& -\frac{(b+2 \alpha)(\beta-\gamma \alpha)\left(d \Re_{0 \gamma}+b\right)}{b+d} \\
& +\frac{b(c+b+d+2 \alpha)(\beta-\gamma \alpha)}{b+d} \\
& -\frac{2 b(c+b+d+2 \alpha)(\beta-\gamma \alpha)}{(b+d) \Re_{0 \gamma}} \\
& +\frac{b(c+b+d+2 \alpha)(\beta-\gamma \alpha)}{(b+d) \mathfrak{R}_{0 \gamma}^{2}} \\
> & \frac{\left[b d+b^{2}+2 d \alpha+b \beta+b \alpha(2-\gamma)\right](c+b+d+2 \alpha)}{b+d} \\
& -\frac{(b+2 \alpha)(\beta-\gamma \alpha)\left(d \mathfrak{R}_{0 \gamma}+b\right)}{b+d} \\
& -\frac{b(c+b+d+2 \alpha)(\beta-\gamma \alpha)}{(b+d) \mathfrak{R}_{0 \gamma}^{2}}
\end{aligned}
$$

Since $0 \leq \gamma \leq 1$, clearly we have $J_{1}>0$. The same to above analysis, for $J_{3}$, we also obtain that

$$
\begin{aligned}
J_{3}= & a_{22} a_{33}-a_{23} a_{32} \\
= & -\frac{(b+2 \alpha)(\beta-\gamma \alpha)\left(d \Re_{0 \gamma}+b\right)}{(b+d) \mathfrak{R}_{0 \gamma}^{2}} \\
& +(b+2 \alpha)(c+b+d+2 \alpha) \\
& +\frac{b d(\beta-\gamma \alpha)\left(\mathfrak{R}_{0 \gamma}-1\right)}{(b+d) \mathfrak{R}_{0 \gamma}^{2}} .
\end{aligned}
$$

$J_{3}>0$ can be shown as the following two cases.

Case $1(\beta-\gamma \alpha>0)$. Consider

$$
\begin{aligned}
J_{3}> & (b+2 \alpha)\left[2 \alpha+(\beta-\gamma \alpha+2 \gamma \alpha) \frac{1}{\mathfrak{R}_{0 \gamma}}\right] \\
& -(b+2 \alpha)(\beta-\gamma \alpha) \frac{1}{\mathfrak{R}_{0 \gamma}}+\frac{b d(\beta-\gamma \alpha)\left(\mathfrak{R}_{0 \gamma}-1\right)}{(b+d) \mathfrak{R}_{0 \gamma}^{2}} \\
= & (b+2 \alpha)\left(2 \alpha+2 \gamma \alpha \frac{1}{\mathfrak{R}_{0 \gamma}}\right)+\frac{b d(\beta-\gamma \alpha)\left(\mathfrak{R}_{0 \gamma}-1\right)}{(b+d) \mathfrak{R}_{0 \gamma}^{2}} .
\end{aligned}
$$

Case $2(\beta-\gamma \alpha \leq 0)$. Consider

$$
\begin{aligned}
J_{3}= & -(\beta-\gamma \alpha) \frac{b d \Re_{0 \gamma}+b^{2}+2 d \alpha \mathfrak{R}_{0 \gamma}+2 b \alpha-b d \mathfrak{R}_{0 \gamma}+b d}{(b+d) \mathfrak{R}_{0 \gamma}^{2}} \\
& +(b+2 \alpha)(c+b+d+2 \alpha) \\
= & \frac{-(\beta-\gamma \alpha)\left(b^{2}+2 d \alpha \Re_{0 \gamma}+2 b \alpha+b d\right)}{(b+d) \mathfrak{R}_{0 \gamma}^{2}} \\
& +(b+2 \alpha)(c+b+d+2 \alpha)>0 .
\end{aligned}
$$

(iii) $A_{3}>0, A_{1} A_{2}-A_{3}>0$

$$
\begin{aligned}
A_{3}= & -\operatorname{det}(A-B) \\
= & -\left(a_{11} a_{22} a_{33}+a_{13} a_{21} a_{32}\right. \\
& \left.\quad-a_{12} a_{21} a_{33}-a_{11} a_{23} a_{32}\right) \\
= & (b+2 \alpha) J_{3}+\frac{b(\beta-\gamma \alpha)}{b+d}\left(1-\frac{1}{\mathfrak{R}_{0 \gamma}}\right)^{2} \\
& \times(b+2 \alpha)(c+b+d+2 \alpha) .
\end{aligned}
$$

Case $1(\beta-\gamma \alpha>0)$. Due to $J_{3}>0$, so $A_{3}>0$.

Case $2(\beta-\gamma \alpha \leq 0)$. According to $\mathfrak{R}_{0 \gamma}>1$, we have

$$
\begin{aligned}
A_{3}= & (b+2 \alpha)\left[\frac{-(\beta-\gamma \alpha)\left(b^{2}+2 d \alpha \mathfrak{R}_{0 \gamma}+2 b \alpha+b d\right)}{(b+d) \mathfrak{R}_{0 \gamma}^{2}}\right. \\
& +(b+2 \alpha)(c+b+d+2 \alpha)] \\
& +\frac{b(b+2 \alpha)(c+b+d+2 \alpha)(\beta-\gamma \alpha)}{b+d} \\
& -\frac{2 b(b+2 \alpha)(c+b+d+2 \alpha)(\beta-\gamma \alpha)}{(b+d) \mathfrak{R}_{0 \gamma}} \\
& +\frac{b(b+2 \alpha)(c+b+d+2 \alpha)(\beta-\gamma \alpha)}{(b+d) \mathfrak{R}_{0 \gamma}^{2}} \\
> & -\frac{(\beta-\gamma \alpha)(b+2 \alpha)\left(b^{2}+2 d \alpha \Re_{0 \gamma}+2 b \alpha+b d\right)}{(b+d) \mathfrak{R}_{0 \gamma}^{2}} \\
& +(b+2 \alpha)(c+b+d+2 \alpha)\left[b^{2}+b d+2 d \alpha+b \beta\right. \\
& \left.-\frac{b(b+2 \alpha)(c+b+d+2 \alpha)(\beta-\gamma \alpha)}{(b+d) \mathfrak{R}_{0 \gamma}^{2}}>0 .(2-\gamma) b \alpha\right] \times(b+d)^{-1}
\end{aligned}
$$

Since $\mathfrak{R}_{0 \gamma}>1$, we have $J_{3}>0$. 
Since $0 \leq \gamma \leq 1$, clearly one has $A_{3}>0$. On the other hand,

$$
\begin{aligned}
A_{1} A_{2}-A_{3}= & -\left(a_{11}+a_{22}+a_{33}\right)\left(J_{1}+J_{2}+J_{3}\right)+\operatorname{det}(A-B) \\
= & -a_{11}\left(J_{1}+J_{2}\right)-a_{22}\left(J_{1}+J_{2}+J_{3}\right) \\
& -a_{33}\left(J_{2}+J_{3}\right)+a_{13} a_{21} a_{32}-a_{11} a_{22} a_{33}
\end{aligned}
$$

because $a_{13} a_{21} a_{32}=\left(b^{2} d(\beta-\gamma \alpha)^{2}\left(\Re_{0 \gamma}-1\right) /(b+d)^{2} \Re_{0 \gamma}^{2}\right)(1-$ $\left.\left(1 / \mathfrak{R}_{0 \gamma}\right)\right)^{2}>0$. So $A_{1} A_{2}-A_{3}>0$, since $a_{i i}<0, J_{i}>0$, $i=1,2,3$.

Therefore, $A_{1} A_{2}-A_{3}>0$. By Lemma $2, A-B$ is also stable. Hence $E_{+}$is locally asymptotically stable. This completes the proof.

\section{Permanence}

Firstly, we consider permanence of the disease. Set initial conditions as $S_{i}(0) \geq 0, I_{i}(0) \geq 0$, and $R_{i}(0) \geq 0$ for $i=1,2$. It is easy to check that all solutions of system (2) are nonnegative (i.e., $S_{i}(t) \geq 0, I_{i}(t) \geq 0$, and $R_{i}(t) \geq 0$ for $t \geq 0$ and $i=1,2$ ) under the assumption $0 \leq \gamma \leq 1$. The following result shows that system (2) is ultimately bounded above.

Theorem 4. There exists an $M>0$ such that for any solution $\left(S_{1}(t), I_{1}(t), R_{1}(t), S_{2}(t), I_{2}(t), R_{2}(t)\right)$ of system (2) with initial values $S_{i}(0) \geq 0, I_{i}(0) \geq 0$, and $R_{i}(0) \geq 0, i=1,2$, there must be a $t_{1}>0$ such that $S_{i}(t) \leq M, I_{i}(t) \leq M$, and $R_{i}(t) \leq M$ for $i=1,2$ and $t \geq t_{1}$.

Proof. Let $V(t)=S_{1}(t)+I_{1}(t)+R_{1}(t)+S_{2}(t)+I_{2}(t)+R_{2}(t)$. We have

$$
\begin{aligned}
\dot{V}(t)= & 2 a-b S_{1}-c I_{1}-b I_{1}-b R_{1}-b S_{2}-c I_{2}-b I_{2}-b R_{2} \\
= & 2 a-b S_{1}-(b+c) I_{1}-b R_{1}-b S_{2} \\
& -(b+c) I_{2}-b R_{2} \leq 2 a-b V .
\end{aligned}
$$

Hence, by comparison theory of differential equations, it is easy to verify that there exists $t_{1}>0$ such that $V(t) \leq M \triangleq$ $(2 a / b)+\varepsilon, t \geq t_{1}$ for $\varepsilon>0$. Then $S_{i}(t) \leq V(t) \leq M, I_{i}(t) \leq$ $V(t) \leq M$, and $R_{i}(t) \leq V(t) \leq M$ for $t \geq t_{1}$. This completes the proof.

Theorem 5. Let $\mathfrak{R}_{0 \gamma}>1$. Then there exists an $\varepsilon>0$ such that every solution $\left(S_{1}(t), I_{1}(t), R_{1}(t), S_{2}(t), I_{2}(t), R_{2}(t)\right)$ of system (2) with initial values $S_{i}(0) \geq 0, I_{1}(0)+I_{2}(0)>0$ and $R_{i}(0) \geq 0$ for $i=1,2$ satisfies

$$
\begin{gathered}
\liminf _{t \rightarrow \infty} S_{i}(t) \geq \varepsilon, \quad \liminf _{t \rightarrow \infty} I_{i}(t) \geq \varepsilon \\
\liminf _{t \rightarrow \infty} R_{i}(t) \geq \varepsilon, \quad i=1,2 .
\end{gathered}
$$

Proof. By system (2) and the fact that $0 \leq \gamma \leq 1$, we have

$$
\dot{S}_{i} \geq a-(\beta+b+\alpha) S_{i}, \quad i=1,2 .
$$

Hence, $S_{i}$ is always ultimately lower bounded by some positive constant; see, for example, $m_{s}=a /(2(\beta+b+\alpha))$, which is independent of initial values. And so is $R_{i}$ if both $I_{1}$ and $I_{2}$ are ultimately lower bounded by some positive constant independent of initial values. Therefore, it suffices to prove that $\lim \inf _{t \rightarrow \infty} I_{i}(t) \geq \varepsilon, i=1,2$.

The result follows from an application of Theorem 4.6 in Arino et al. [18]. Define

$$
\begin{gathered}
X=\left\{\left(S_{1}, I_{1}, R_{1}, S_{2}, I_{2}, R_{2}\right) \mid S_{i} \geq 0, I_{i} \geq 0, R_{i} \geq 0, i=1,2\right\}, \\
X_{0}=\left\{\left(S_{1}, I_{1}, R_{1}, S_{2}, I_{2}, R_{2}\right) \in X \mid I_{1}+I_{2}>0, i=1,2\right\}, \\
\partial X_{0}=X \backslash X_{0} .
\end{gathered}
$$

It then suffices to show that system (2) is uniformly persistent with respect to $\left(X_{0}, \partial X_{0}\right)$.

Next, $X$ is positively invariant with respect to system (2). It is easy to verify that $I_{1}(t)+I_{2}(t)>0, i=1,2$ for $t>0$ if $S_{i}(0) \geq 0, R_{i}(0) \geq 0$, and $I_{1}(0)+I_{2}(0)>0$ for $i=1,2$. This $X_{0}$ is also positively invariant. Furthermore, by Theorem 4, there exists a compact set $B$ in which all solutions of system (2) initiated in $X$ will enter and remain forever after. The compactness condition $\left(C_{4.2}\right)$ in Arino et al. [18] is easily verified for this set $B$. Denote

$$
\begin{aligned}
M_{\partial}= & \left\{\left(S_{1}(0), I_{1}(0), R_{1}(0), S_{2}(0), I_{2}(0), R_{2}(0)\right) \mid\right. \\
& \left(S_{1}(t), I_{1}(t), R_{1}(t), S_{2}(t), I_{2}(t), R_{2}(t)\right) \in \partial X_{0}, \\
& \forall t \geq 0\} .
\end{aligned}
$$

We now show that

$$
M_{\partial}=\left\{\left(S_{1}, 0, R_{1}, S_{2}, 0, R_{2}\right) \mid S_{i} \geq 0, R_{i} \geq 0, i=1,2\right\} .
$$

Suppose that $\left(S_{1}(0), I_{1}(0), R_{1}(0), S_{2}(0), I_{2}(0), R_{2}(0)\right) \in M_{\partial}$. It suffices to show $I_{i}(t)=0$ for any $t \geq 0$ and $i=1,2$. If not, there exists $t_{0} \geq 0$ such that $I_{1}\left(t_{0}\right)>0$ or $I_{2}\left(t_{0}\right)>0$. Hence, $I_{1}\left(t_{0}\right)+I_{2}\left(t_{0}\right)>0$ and $\left(S_{1}\left(t_{0}\right), I_{1}\left(t_{0}\right), R_{1}\left(t_{0}\right), S_{2}\left(t_{0}\right)\right.$, $\left.I_{2}\left(t_{0}\right), R_{2}\left(t_{0}\right)\right) \in X_{0}$, which contradicts $\left(S_{1}(0), I_{1}(0), R_{1}(0)\right.$, $\left.S_{2}(0), I_{2}(0), R_{2}(0)\right) \in M_{\partial}$. This proves (38).

Denote the omega limit set of the solutions of system (2) starting in $\left(S_{1}(0), I_{1}(0), R_{1}(0), S_{2}(0), I_{2}(0), R_{2}(0)\right) \in X$ by $\omega\left(S_{1}(0), I_{1}(0), R_{1}(0), S_{2}(0), I_{2}(0), R_{2}(0)\right.$ ) (which exists by Theorem 4). Let

$$
\begin{aligned}
\Omega=\cup\left\{\omega\left(S_{1}(0), I_{1}(0), R_{1}(0), S_{2}(0), I_{2}(0), R_{2}(0)\right) \mid\right. \\
\left.\left(S_{1}(0), I_{1}(0), R_{1}(0), S_{2}(0), I_{2}(0), R_{2}(0)\right) \in M_{\partial}\right\} .
\end{aligned}
$$

Restricting system (2) on $M_{\partial}$ gives

$$
\begin{aligned}
& \dot{S}_{1}=a-(b+\alpha) S_{1}+\alpha S_{2} \\
& \dot{R}_{1}=-(b+\alpha) R_{1}+\alpha R_{2} \\
& \dot{S}_{2}=a-(b+\alpha) S_{2}+\alpha S_{1} \\
& \dot{R}_{2}=-(b+\alpha) R_{2}+\alpha R_{1} .
\end{aligned}
$$


It is easy to verify that system (40) has a unique equilibrium $E_{1}\left(S^{0}, 0, S^{0}, 0\right)$, where $S^{0}=a / b$. And thus $E_{0}\left(S^{0}, 0,0, S^{0}, 0,0\right)$ is the unique equilibrium of system (2) in $M_{\partial}$. It is easy to check that $E_{1}$ is locally asymptotically stable. Hence, it is also globally asymptotically stable since system (40) is a linear system. Thus $\Omega=\left\{E_{0}\right\}$. And $E_{0}$ is a covering of $\Omega$, which is isolated (since $E_{0}$ is the unique equilibrium) and is acyclic (since there exists no solution in $M_{\partial}$ which links $E_{0}$ to itself). Finally, the proof will be done if we show that $E_{0}$ is a weak repeller for $X_{0}$; that is,

$$
\limsup _{t \rightarrow \infty} \operatorname{dist}\left(\left(S_{1}(t), I_{1}(t), R_{1}(t), S_{2}(t), I_{2}(t), R_{2}(t)\right), E_{0}\right)>0,
$$

where $\left(S_{1}(t), I_{1}(t), R_{1}(t), S_{2}(t), I_{2}(t), R_{2}(t)\right)$ is the solution of system (2) with initial value $\left(S_{1}(0), I_{1}(0), R_{1}(0), S_{2}(0)\right.$, $\left.I_{2}(0), R_{2}(0)\right) \in X_{0}$. By the proof of Lemma 3.5 in Cui et al. [19], (41) is valid if

$$
W^{S}\left(E_{0}\right) \cap X_{0}=\phi \text {, }
$$

where $W^{s}\left(E_{0}\right)$ denotes the stable manifold of $E_{0}$. Suppose (42) is not valid; then there exists a solution $\left(S_{1}(t)\right.$, $\left.I_{1}(t), R_{1}(t), S_{2}(t), I_{2}(t), R_{2}(t)\right) \in X_{0}, t \geq 0$ of system (2) with initial value $\left(S_{1}(0), I_{1}(0), R_{1}(0), S_{2}(0), I_{2}(0), R_{2}(0)\right) \in X_{0}$, such that

$$
\begin{array}{r}
S_{i}(t) \longrightarrow S^{0}, \quad I_{i}(t) \longrightarrow 0, \quad R_{i}(t) \longrightarrow 0 \\
\text { as } t \longrightarrow \infty, \quad i=1,2 .
\end{array}
$$

Since $\mathfrak{R}_{0 \gamma}=(\beta+\gamma \alpha) /(b+d+c)>1$, we can choose $\delta>0$ which is small enough such that $S^{0}-\delta>0$ and

$$
m_{\delta} \triangleq \frac{(\beta+\gamma \alpha)\left(S^{0}-\delta\right)}{S^{0}}-(b+d+c)>0 .
$$

Define $V=I_{1}+I_{2}$. For $S^{0}-\delta>0$, by (43) there exists $t_{1}>0$ such that

$$
\begin{array}{r}
S^{0}-\delta<S_{i}(t)<S^{0}+\delta, \quad 0<I_{i}(t)<\delta, \quad 0<R_{i}(t)<\delta, \\
t \geq t_{1}, \quad i=1,2 .
\end{array}
$$

Hence by system (2),

$$
\begin{aligned}
\dot{V}= & (\beta+\gamma \alpha)\left(\frac{S_{1} I_{1}}{S_{1}+I_{1}+R_{1}}+\frac{S_{2} I_{2}}{S_{2}+I_{2}+R_{2}}\right) \\
& -(c+b+d)\left(I_{1}+I_{2}\right) \\
\geq & (\beta+\gamma \alpha)\left(\frac{S^{0}-\delta}{S^{0}-\delta+\delta} I_{1}+\frac{S^{0}-\delta}{S^{0}-\delta+\delta} I_{2}\right) \\
& -(c+d+b)\left(I_{1}+I_{2}\right) \\
= & m_{\delta} V,
\end{aligned}
$$

for $t \geq t_{1}$. Hence, $V(t) \geq V\left(t_{1}\right) \exp \left(m_{\delta}\left(t-t_{1}\right)\right)$. By (44), we have $V(t) \rightarrow \infty$ as $t \rightarrow \infty$, which contradicts (43). Thus (42) holds, which completes the proof.

\section{Global Stability}

First of all, we will discuss the global stability of $E_{0}$ under the condition $\mathfrak{R}_{0 \gamma} \leq 1$.

Theorem 6. If $\mathfrak{R}_{0 \gamma} \leq 1$, then $E_{0}$ is globally asymptotically stable.

Proof. Consider the following function:

$$
V(t)=I_{1}+I_{2} \text {. }
$$

Its derivative along the solutions of system (2) is

$$
\begin{aligned}
\dot{V}(t)= & (\beta+\gamma \alpha)\left(\frac{S_{1} I_{1}}{S_{1}+I_{1}+R_{1}}+\frac{S_{2} I_{2}}{S_{2}+I_{2}+R_{2}}\right) \\
& -(c+b+d)\left(I_{1}+I_{2}\right) .
\end{aligned}
$$

If $\mathfrak{R}_{0 \gamma}<1$, then

$$
\dot{V}(t) \leq[(\beta+\gamma \alpha)-(c+b+d)]\left(I_{1}+I_{2}\right) \leq 0 .
$$

If $\mathfrak{R}_{0 \gamma}=1$, then

$$
\dot{V}(t)=-(\beta+\gamma \alpha)\left[\frac{I_{1}\left(I_{1}+R_{1}\right)}{S_{1}+I_{1}+R_{1}}+\frac{I_{2}\left(I_{2}+R_{2}\right)}{S_{2}+I_{2}+R_{2}}\right] \leq 0 .
$$

When $\mathfrak{R}_{0 \gamma} \leq 1$, we set

$$
\begin{aligned}
L & =\left\{\left(S_{1}, I_{1}, R_{1}, S_{2}, I_{2}, R_{2}\right) \mid \dot{V}(t)=0\right\} \\
& =\left\{\left(S_{1}, I_{1}, R_{1}, S_{2}, I_{2}, R_{2}\right) \mid I_{1}=0, I_{2}=0\right\} .
\end{aligned}
$$

Restricting system (2) on the set $L$, we have $d\left(R_{1}+R_{2}\right) / d t=$ $-b\left(R_{1}+R_{2}\right)$, then $\lim _{t \rightarrow \infty} R_{i}(t)=0$ for $i=1,2 . d\left(S_{1}+S_{2}\right) / d t=$ $2 a-b\left(S_{1}+S_{2}\right)$, then $\lim _{t \rightarrow \infty} S_{i}(t)=a / b$ for $i=1,2$. Therefore, $M=\left\{E_{0}\right\}$ is the largest positively invariant subset of $L$. By Lyapunov-LaSalle theorem, $E_{0}$ is global asymptotically stable provided $\mathfrak{R}_{0 \gamma} \leq 1$. This completes the proof.

Theorems 4 and 5 imply that system (2) is permanent if $\mathfrak{R}_{0 \gamma}>1$. Next, we consider the global asymptotic stability of $E_{+}$. The set $X_{0}$ defined in proof of Theorem 5 will be used.

Theorem 7. Suppose that

$$
b+2 \alpha>\max \left\{\frac{9}{2}|\beta-\gamma \alpha|, \frac{d}{2}+|\beta-\gamma \alpha|\right\} .
$$

Then the endemic equilibrium point $E_{+}$is globally asymptotically stable on $X_{0}$ for $\mathfrak{R}_{0 \gamma}>1$.

Proof. By Theorem let us consider the function:

$$
\begin{gathered}
V(t)=\left\{\left(S_{1}(t)-S_{2}(t)\right)^{2}+\left(I_{1}(t)-I_{2}(t)\right)^{2}\right. \\
\left.+\left(R_{1}(t)-R_{2}(t)\right)^{2}\right\} \times(2)^{-1} .
\end{gathered}
$$


The time derivative of $V(t)$ along the solution of system (2) becomes

$$
\begin{aligned}
\dot{V}= & \left(\dot{S}_{1}-\dot{S}_{2}\right)\left(S_{1}-S_{2}\right)+\left(\dot{I}_{1}-\dot{I}_{2}\right)\left(I_{1}-I_{2}\right) \\
& +\left(\dot{R}_{1}-\dot{R}_{2}\right)\left(R_{1}-R_{2}\right) \\
= & \left\{(\beta-\gamma \alpha)\left(\frac{S_{2} I_{2}}{S_{2}+R_{2}+I_{2}}-\frac{S_{1} I_{1}}{S_{1}+I_{1}+R_{1}}\right)\right. \\
& \left.-(b+2 \alpha)\left(S_{1}-S_{2}\right)\right\}\left(S_{1}-S_{2}\right) \\
& +\left\{(\beta-\gamma \alpha)\left(\frac{S_{1} I_{1}}{S_{1}+I_{1}+R_{1}}-\frac{S_{2} I_{2}}{S_{2}+R_{2}+I_{2}}\right)\right. \\
& \left.\quad-(c+b+d+2 \alpha)\left(I_{1}-I_{2}\right)\right\}\left(I_{1}-I_{2}\right) \\
+ & \left\{d\left(I_{1}-I_{2}\right)-(b+2 \alpha)\left(R_{1}-R_{2}\right)\right\}\left(R_{1}-R_{2}\right) .
\end{aligned}
$$

Note that

$$
\begin{aligned}
& \frac{S_{2} I_{2}}{S_{2}+I_{2}+R_{2}}-\frac{S_{1} I_{1}}{S_{1}+I_{1}+R_{1}} \\
& \quad=\frac{S_{1} S_{2} I_{2}+S_{2} I_{1} I_{2}+S_{2} I_{2} R_{1}-S_{1} I_{1} S_{2}-S_{1} I_{1} S_{2}-S_{1} I_{1} R_{2}}{\left(S_{1}+I_{1}+R_{1}\right)\left(S_{2}+I_{2}+R_{2}\right)} \\
& \quad \leq 2\left|I_{2}-I_{1}\right|+2\left|S_{2}-S_{1}\right|+\left|R_{1}-R_{2}\right|,
\end{aligned}
$$

which gives the following:

$$
\begin{aligned}
\dot{V} \leq & -(b+2 \alpha)\left(S_{1}-S_{2}\right)^{2}-(c+2 \alpha+b+d)\left(I_{1}-I_{2}\right)^{2} \\
& -(b+2 \alpha)\left(R_{1}-R_{2}\right)^{2}+d\left(I_{1}-I_{2}\right)\left(R_{1}-R_{2}\right) \\
& +(\beta-\gamma \alpha)\left(\frac{S_{2} I_{2}}{S_{2}+I_{2}+R_{2}}-\frac{S_{1} I_{1}}{S_{1}+I_{1}+R_{1}}\right) \\
& \times\left(S_{1}-S_{2}-I_{1}+I_{2}\right) \\
\leq & -(b+2 \alpha)\left(S_{1}-S_{2}\right)^{2}-(c+2 \alpha+b+d)\left(I_{1}-I_{2}\right)^{2} \\
& -(b+2 \alpha)\left(R_{1}-R_{2}\right)^{2}+d\left|I_{1}-I_{2}\right|\left|R_{1}-R_{2}\right| \\
& +|\beta-\gamma \alpha|\left(2\left|I_{1}-I_{2}\right|+2\left|S_{1}-S_{2}\right|+\left|R_{1}-R_{2}\right|\right) \\
& \times\left(\left|S_{1}-S_{2}\right|+\left|I_{1}-I_{2}\right|\right) \\
\leq & -\left\{(b+2 \alpha)-\frac{9}{2}|\beta-\gamma \alpha|\right\}\left|S_{1}-S_{2}\right|^{2} \\
& -\left\{\left(c+2 \alpha+b+\frac{1}{2} d\right)-\frac{9}{2}|\beta-\gamma \alpha|\right\}\left|I_{1}-I_{2}\right|^{2} \\
& -\left\{(b+2 \alpha)-|\beta-\gamma \alpha|-\frac{1}{2} d\right\}\left|R_{1}-R_{2}\right|^{2} .
\end{aligned}
$$

The above quadratic form is negative definite if and only if

$$
b+2 \alpha>\frac{9}{2}|\beta-\gamma \alpha|, \quad b+2 \alpha>\frac{d}{2}+|\beta-\gamma \alpha| .
$$

It is easy to check that the above conditions are satisfied if and only if (52) is satisfied. Hence we can find some positive constant $\lambda=\min \{b+2 \alpha-(9 / 2)|\beta-\gamma \alpha|, b+2 \alpha-(d / 2)-|\beta-\gamma \alpha|\}$ satisfying

$$
\dot{V} \leq \lambda\left\{\left(S_{1}-S_{2}\right)^{2}+\left(I_{1}-I_{2}\right)^{2}+\left(R_{1}-R_{2}\right)^{2}\right\}=-2 \lambda V(t),
$$

which shows that for any solution $\left(S_{1}(t), I_{1}(t), R_{1}(t), S_{2}(t)\right.$, $I_{2}(t), R_{2}(t)$ ) of system (2), we have

$$
\begin{aligned}
& \lim _{t \rightarrow \infty}\left\{S_{1}(t)-S_{2}(t)\right\}=0, \\
& \lim _{t \rightarrow \infty}\left\{I_{1}(t)-I_{2}(t)\right\}=0, \\
& \lim _{t \rightarrow \infty}\left\{R_{1}(t)-R_{2}(t)\right\}=0 .
\end{aligned}
$$

By Lyapunov's theorem, we know that $\omega(x) \cap \mathbb{R}_{+}^{6}$ is contained in the set $\left\{x \in \mathbb{R}_{+}^{6} \mid \dot{V}=0\right\}=\left\{\left(S_{1}, I_{1}, R_{1}, S_{2}, I_{2}, R_{2}\right) \in\right.$ $\left.\mathbb{R}_{+}^{6} \mid S_{1}=S_{2}, I_{1}=I_{2}, R_{1}=R_{2}\right\}$. Here $\omega(x)$ is the $\omega$-limit set of the solution of system (2) with an initial value $x$ and $\mathbb{R}_{+}^{6}$ is the state space $\left\{x \in \mathbb{R}^{6} \mid x \geq 0\right\}$. On the set $\Omega=\left\{\left(S_{1}, I_{1}\right.\right.$, $\left.\left.R_{1}, S_{2}, I_{2}, R_{2}\right) \in \mathbb{R}_{+}^{6} \mid S_{1}=S_{2}, I_{1}=I_{2}, R_{1}=R_{2}\right\}$, we now consider the following system for $S=S_{i}, I=I_{i}$, and $R=$ $R_{i}(i=1,2)$; that is,

$$
\begin{gathered}
\dot{S}=a-b S-\frac{(\beta+\gamma \alpha) S I}{S+I+R}=f(S, I, R), \\
\dot{I}=\frac{(\beta+\gamma \alpha) S I}{S+I+R}-(c+b+d) I=g(S, I, R), \\
\dot{R}=d I-b R=h(S, I, R) .
\end{gathered}
$$

It is trivial that the equilibrium $\left(S^{*}, I^{*}, R^{*}\right)$ of system $(60)$ is globally asymptotically stable and the equilibrium $(a / b, 0,0)$ is unstable as $\mathfrak{R}_{0 \gamma}>1$. This shows that the endemic equilibrium $E_{+}$of system (2) is globally asymptotically stable. This completes the proof.

\section{Numerical Simulations and Discussion}

In this paper, a two-city SIR epidemic model with transportrelated infections is proposed. According to Theorems 1 and 3 , we obtain that there exist a disease-free equilibrium and an endemic equilibrium which are locally asymptotically stable if the basic reproduction number $\mathfrak{R}_{0 \gamma} \leq 1$ and $\mathfrak{R}_{0 \gamma}>1$, respectively. Theorems 4 and 5 provide the permanence of this SIR model. In addition, sufficient conditions are established in Theorems 6 and 7 for global asymptotic stability of the disease-free and the endemic equilibrium, severally. The following numerical simulations, we will present, are to explain the feasibility of our main results. 

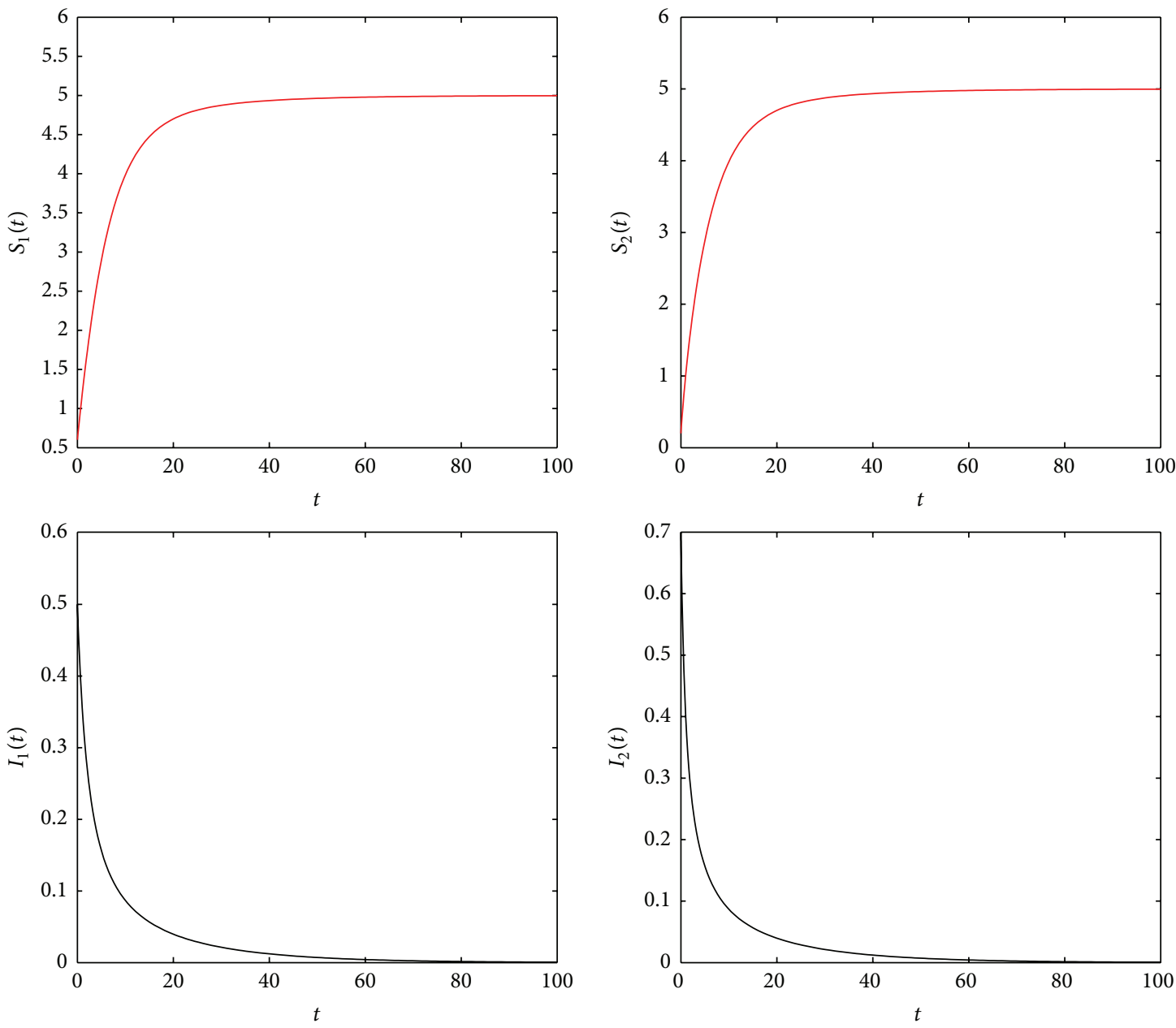

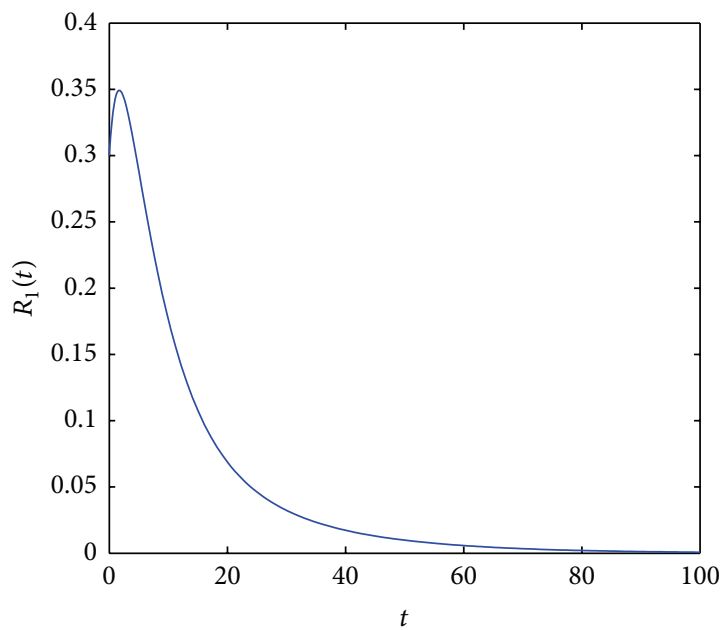

(a)

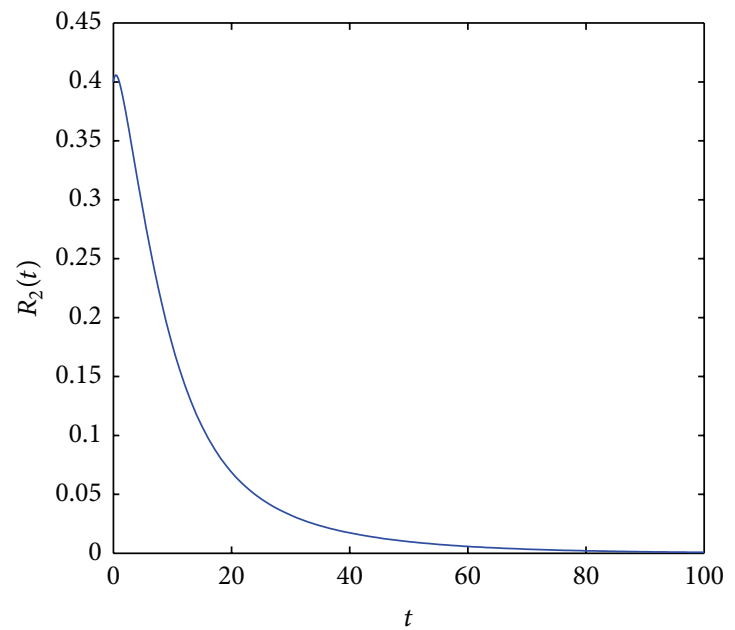

(b)

Figure 1: (a) shows movement paths of $S_{1}, I_{1}$, and $R_{1}$ as functions of time $t$. (b) shows movement paths of $S_{2}, I_{2}$, and $R_{2}$ as functions of time $t$. Here $a=1, b=0.2, c=0.4, d=0.2, \alpha=0.3, \beta=0.6$, and $\gamma=0.5$. Initial data are $0.6,0.5,0.3,0.2,0.7,0.4$.

If we set $a=1, b=0.2, c=0.4, d=0.2, \alpha=0.3, \beta=0.6$, and $\gamma=0.5$, by a simple computation, we derive

$$
\mathfrak{R}_{0 \gamma}=0.9375<1 .
$$

Obviously, the assumptions of Theorems 1 and 6 are satisfied, so disease-free equilibrium of system (2) is globally asymptotically stable. From Figure 1, it is easy to observe that if $\alpha$ is relatively small, then both isolated cities are disease free and 

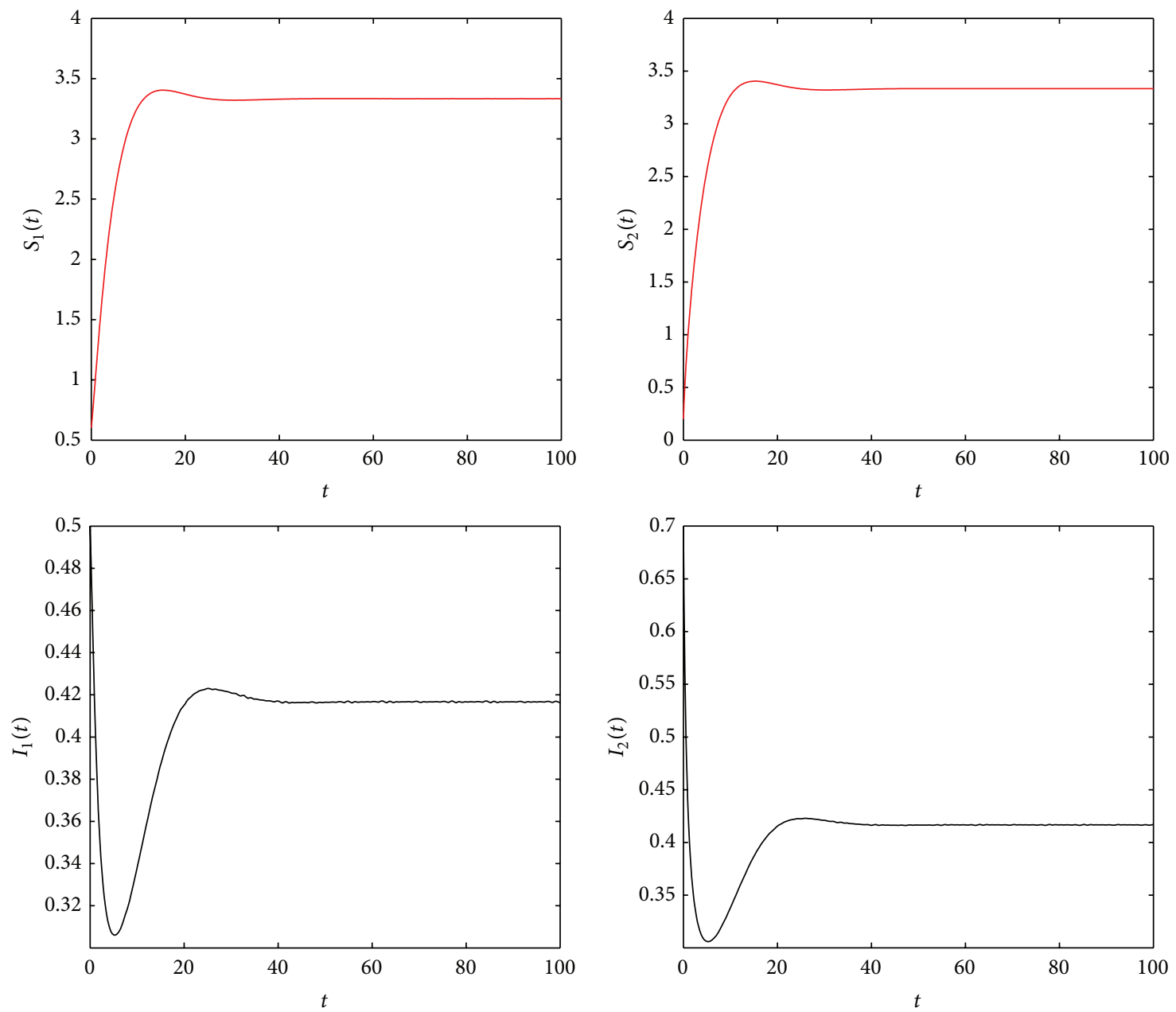

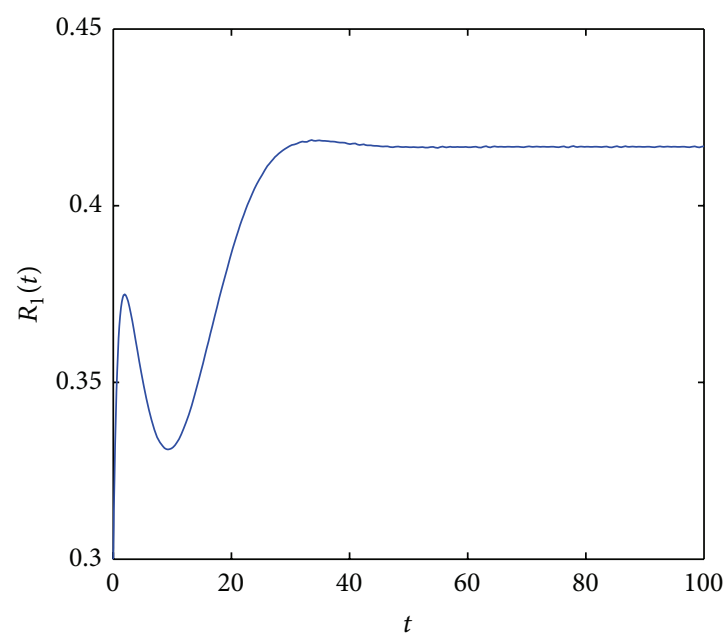

(a)

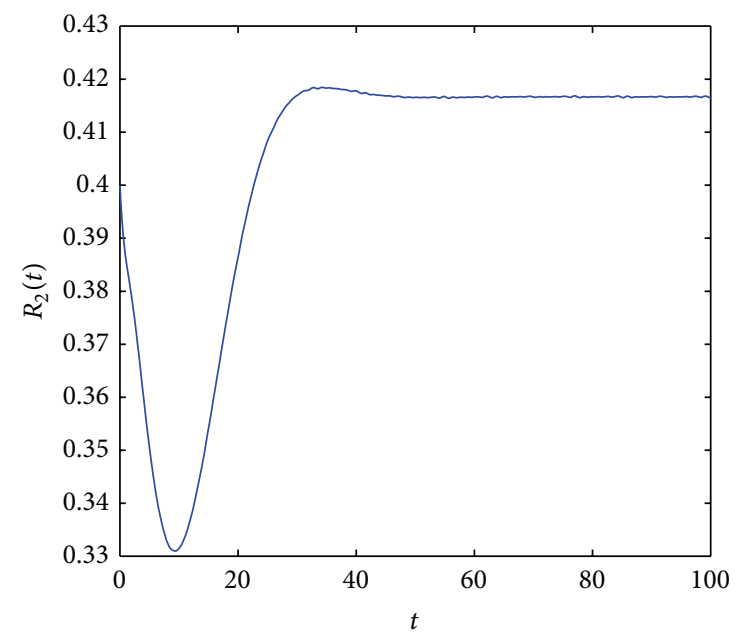

(b)

Figure 2: (a) shows movement paths of $S_{1}, I_{1}$, and $R_{1}$ as functions of time $t$. (b) shows movement paths of $S_{2}, I_{2}$, and $R_{2}$ as functions of time $t$. Here $a=1, b=0.2, c=0.4, d=0.2, \alpha=0.8, \beta=0.6$, and $\gamma=0.5$. Initial data are $0.6,0.5,0.3,0.2,0.7,0.4$. 
the transport-related infection may not lead to the disease becoming endemic.

On the other hand, if we have $a=1, b=0.2, c=0.4$, $d=0.2, \alpha=0.3, \beta=0.6$, and $\gamma=0.5$, we have

$$
\mathfrak{R}_{0 \gamma}=1.2500>1 \text {. }
$$

The assumption of Theorem 3 is satisfied, and since

$$
b+2 \alpha-\max \left\{\frac{9}{2}|\beta-\gamma \alpha|, \frac{d}{2}+|\beta-\gamma \alpha|\right\}=0.9000>0 \text {, }
$$

it is not difficult to prove Theorem 7. Hence, the endemic equilibrium of system (2) is globally asymptotically stable. Figure 2 shows that if $\alpha$ is relatively large, then the disease is endemic in the two isolated cities and the transport-related infection will surely lead to the disease becoming endemic.

\section{Conflict of Interests}

The authors declare that there is no conflict of interests regarding the publication of this paper.

\section{Acknowledgments}

This work is supported by Key Laboratory of Biologic Resources Protection and Utilization of Hubei Province (PKLHB1302) and the soft science research project of Hubei Province (2012GDA01309) and the key discipline of Hubei province-Forestry.

\section{References}

[1] W. O. Kermark and A. G. Mckendrick, "Contributions to the mathematical theory of epidemics," Proceedings of the Royal Society A, vol. 115, no. 772, pp. 700-721, 1927.

[2] Z. Y. Xiang, Y. F. Li, and X. Y. Song, "Dynamic analysis of a pest management SEI model with saturation incidence concerning impulsive control strategy," Nonlinear Analysis: Real World Applications, vol. 10, no. 4, pp. 2335-2345, 2009.

[3] P. D. O’Neill and C. H. Wen, "Modelling and inference for epidemic models featuring non-linear infection pressure," Mathematical Biosciences, vol. 238, no. 1, pp. 38-48, 2012.

[4] C. H. Li, C. C. Tsai, and S. Y. Yang, "Analysis of the permanence of an SIR epidemic model with logistic process and distributed time delay," Communications in Nonlinear Science and Numerical Simulation, vol. 17, no. 9, pp. 3696-3707, 2012.

[5] Z. X. Han and J. D. Zhao, "Stochastic SIRS model under regime switching," Nonlinear Analysis: Real World Applications, vol. 14, no. 1, pp. 352-364, 2013.

[6] L. Zhou, Y. Ma, X. Zhang, and Y. Zhou, "Global analysis of discrete HIV/AIDS models with age-structure for recurrent event data," Acta Mathematicae Applicatae Sinica, vol. 33, no. 3, pp. 466-478, 2010.

[7] I. H. I. Ahmed, P. J. Witbooi, and K. Patidar, "Modeling the dynamics of an epidemic under vaccination in two interacting populations," Journal of Applied Mathematics, vol. 2012, Article ID 275902, 14 pages, 2012.
[8] W. Wang and S. Ruan, "Simulating the SARS outbreak in Beijing with limited data," Journal of Theoretical Biology, vol. 227, no. 3, pp. 369-379, 2004.

[9] D. Q. Ding, X. P. Wang, and X. H. Ding, "Global stability of multigroup dengue disease transmission model," Journal of Applied Mathematics, vol. 2012, Article ID 342472, 11 pages, 2012.

[10] L. Sattenspiel and K. Dietz, "A structured epidemic model incorporating geographic mobility among regions," Mathematical Biosciences, vol. 128, no. 1-2, pp. 71-91, 1995.

[11] L. Sattenspiel and D. A. Herring, "Structured epidemic models and the spread of influenza in the central Canadian subarctic," Human Biology, vol. 70, no. 1, pp. 91-115, 1998.

[12] W. D. Wang and G. Mulone, "Threshold of disease transmission on a patch environment," Journal of Mathematical Analysis and Applications, vol. 285, no. 1, pp. 321-335, 2003.

[13] W. D. Wang and X. Q. Zhao, "An epidemic model in a patchy environment," Mathematical Biosciences, vol. 190, no. 1, pp. 97$112,2004$.

[14] W. D. Wang and X.-Q. Zhao, "An age-structured epidemic model in a patchy environment," SIAM Journal on Applied Mathematics, vol. 65, no. 5, pp. 1597-1614, 2005.

[15] L. J. S. Allen, B. M. Bolker, Y. Lou, and A. L. Nevai, "Asymptotic profiles of the steady states for an SIS epidemic patch model," SIAM Journal on Applied Mathematics, vol. 67, no. 5, pp. 12831309, 2007.

[16] G. H. Li, W. D. Wang, and Z. Jin, "Global stability of an SEIR epidemic model with constant immigration," Chaos, Solitons and Fractals, vol. 30, no. 4, pp. 1012-1019, 2006.

[17] H. L. Liu, H. B. Xu, J. Y. Yu, and G. T. Zhu, "Stability on coupling SIR epidemic model with vaccination," Journal of Applied Mathematics, no. 4, pp. 301-319, 2005.

[18] J. Arino, R. Jordan, and P. van den Driessche, "Quarantine in a multi-species epidemic model with spatial dynamics," Mathematical Biosciences, vol. 206, no. 1, pp. 46-60, 2007.

[19] J. A. Cui, Y. Takeuchi, and Y. Saito, "Spreading disease with transport-related infection," Journal of Theoretical Biology, vol. 239, no. 3, pp. 376-390, 2006. 


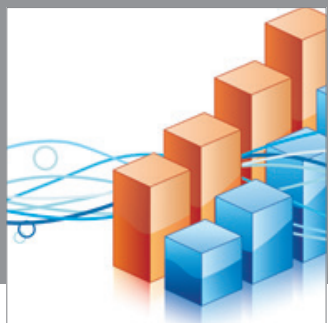

Advances in

Operations Research

mansans

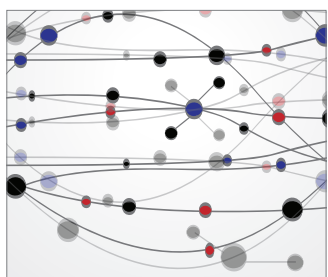

The Scientific World Journal
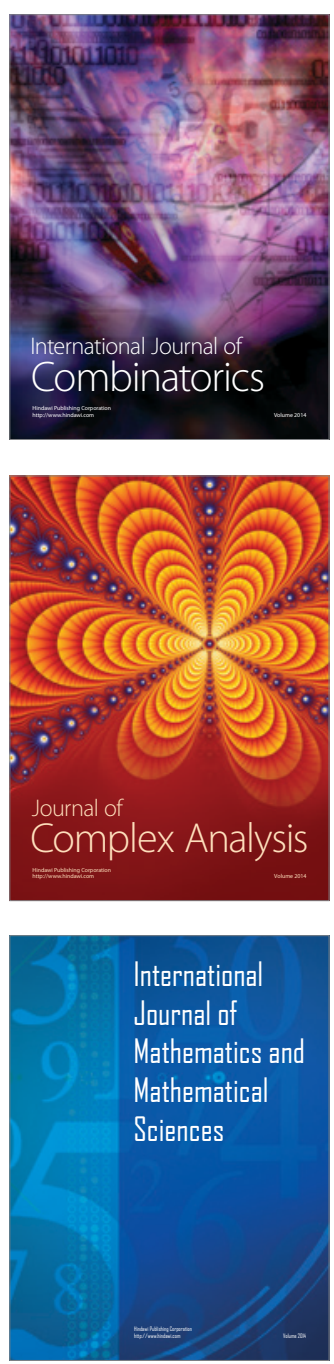
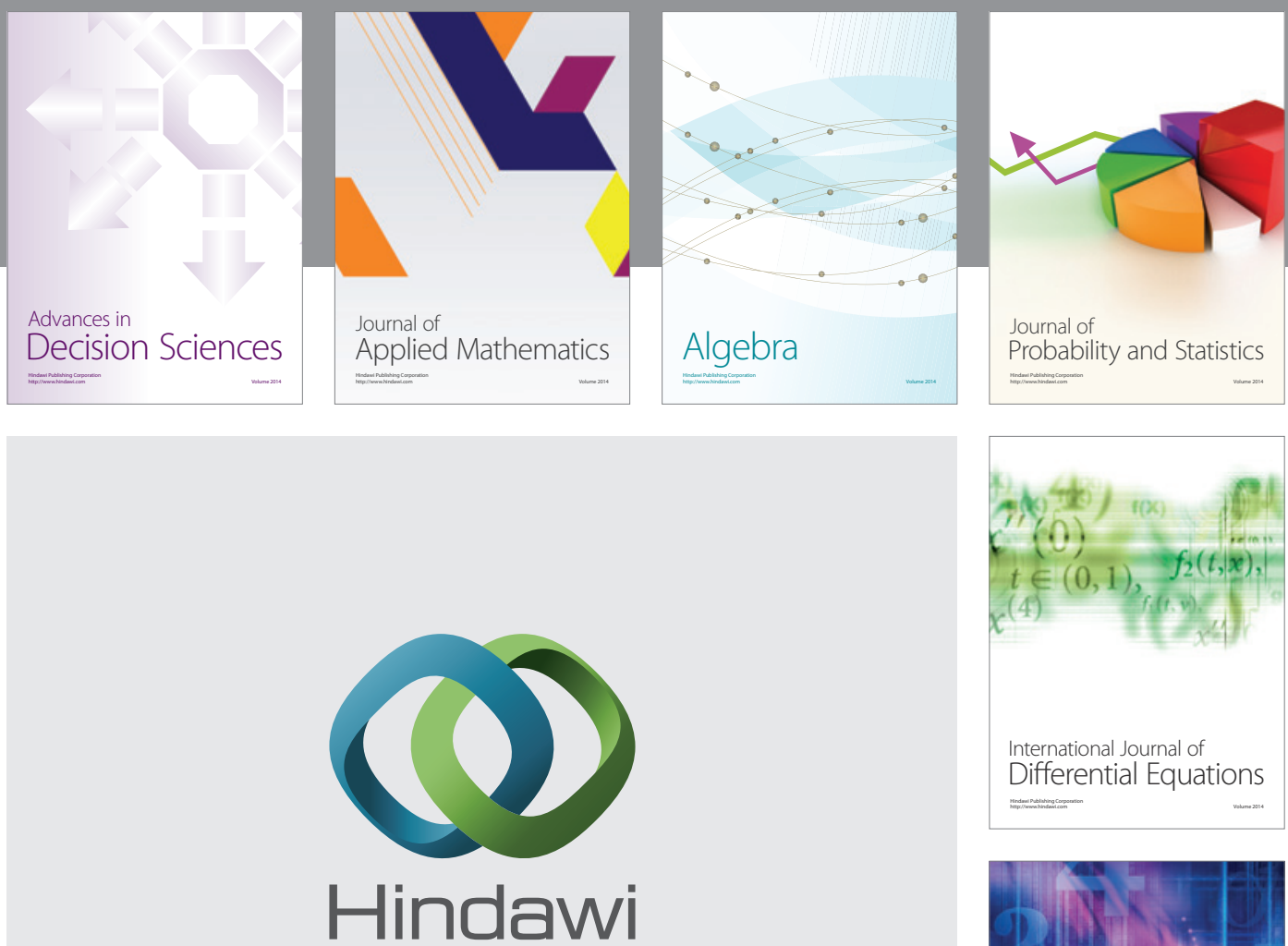

Submit your manuscripts at http://www.hindawi.com
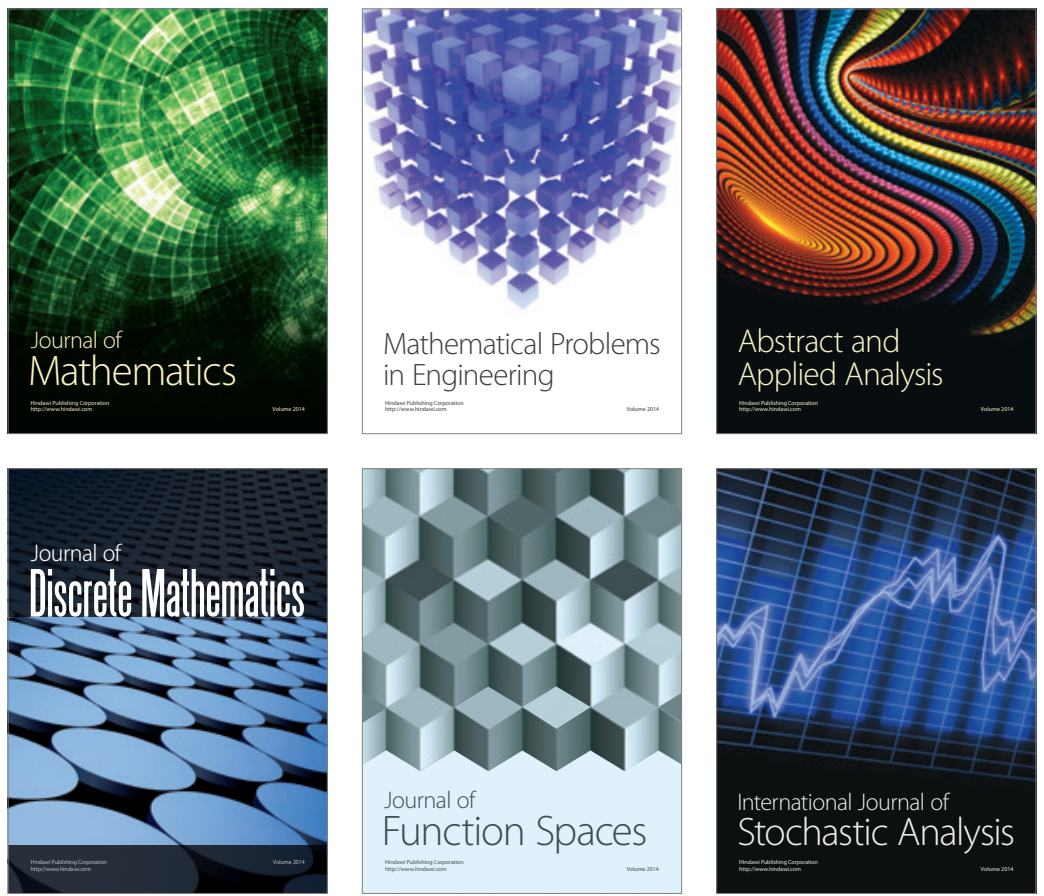

Journal of

Function Spaces

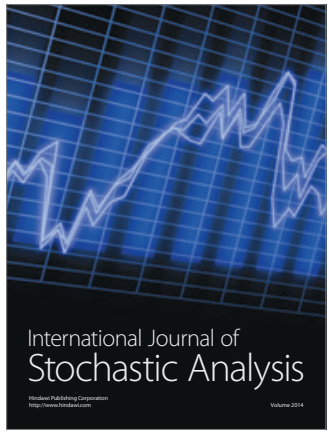

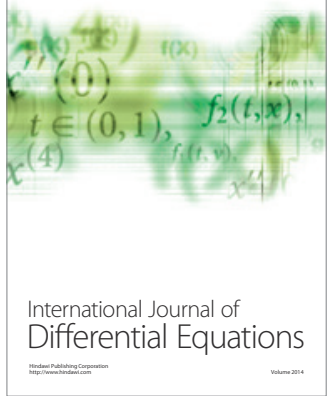
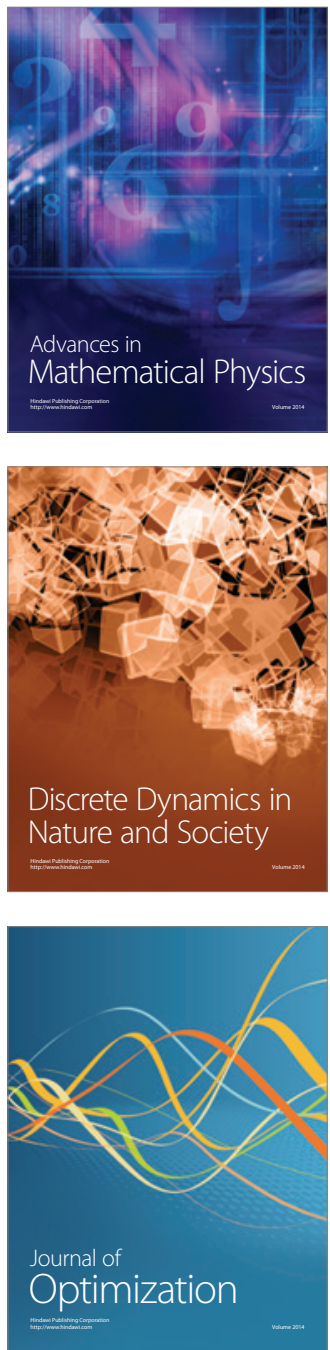